\title{
EXACT COVERING SYSTEMS AND THE GAUSS-LEGENDRE MULTIPLICATION FORMULA FOR THE GAMMA FUNCTION
}

\author{
JOHN BEEBEE
}

(Communicated by William Adams)

\begin{abstract}
The Gauss-Legendre multiplication formula for the gamma function is $(2 \pi)^{(m-1) / 2} m^{1 / 2-m z} \Gamma(m z)=\Gamma(z) \Gamma\left(z+\frac{1}{m}\right) \cdots \Gamma\left(z+\frac{m-1}{m}\right)$. Let $\left\{a_{i}\right.$ $\left.\left(\bmod b_{i}\right): 1 \leq i \leq m\right\}$ be an exact covering system with standardized offsets. Then

$$
\Gamma(z)=\frac{\Gamma\left(z / b_{1}\right)}{b_{1}{ }^{1-z / b_{1}}} \prod_{i=2}^{m} \frac{\Gamma\left(\left(z+a_{i}\right) / b_{i}\right)}{b_{i}^{-z / b_{i}} \Gamma\left(a_{i} / b_{i}\right)} .
$$

Conversely, if the above identity holds, then $\left\{a_{i}\left(\bmod b_{i}\right): 1 \leq i \leq m\right\}$ is an exact covering system with standardized offsets. The Gauss-Legendre multiplication formula is a special case of this identity.
\end{abstract}

Let $Z_{a b}$ be the arithmetic progression (AP) $\{x: x=a+n b, n \in Z\}$. Another notation for this arithmetic progression is $a(\bmod b)$. A finite collection of disjoint AP's $C=\left\{Z_{a_{i} b_{i}}: 1 \leq i \leq m\right\}$ is called an exact covering system (or exact cover) if each integer belongs to exactly one AP $Z_{a_{i} b_{i}}$. We usually assume that the offsets $a_{i}$ have been standardized so that $0 \leq a_{i}<b_{i}$. A consequence of the fact that $C$ is an exact cover with standardized offsets is that there is one and only one offset that is zero, and we assume it is always $a_{1}$. Another property of exact covers is that $\sum_{i=1}^{m} 1 / b_{i}=1$. If the offsets are standardized, $\sum_{i=1}^{m} a_{i} / b_{i}=(m-1) / 2$, by Theorem 1 of Fraenkel [1]. Let $M=\operatorname{lcm}\left\{b_{i}\right\}$. If each of the integers $\{0,1, \ldots, M-1\}$ is covered by $\mathrm{C}$, then all the integers are covered by $\mathrm{C}$. The collection $Z=\left\{Z_{a_{i} b_{i}}: 1 \leq i<\infty\right\}$ is an infinite exact cover if each integer belongs to exactly one AP. There are two classes of infinite exact covers. A saturated cover has $\sum_{i=1}^{\infty} 1 / b_{i}=1$. An unsaturated cover has $\sum_{i=1}^{\infty} 1 / b_{i}<1$.

In [2] I proved that

$$
\sin (\pi z)=b_{1} \sin \left(\frac{\pi z}{b_{1}}\right) \prod_{i=2}^{m} \frac{\sin \pi\left(\left(a_{i}-z\right) / b_{i}\right)}{\sin \left(\pi a_{i} / b_{i}\right)}
$$

if and only if $C=\left\{Z_{a_{i} b_{i}}: 1 \leq i \leq m\right\}$ is an exact cover. A special case of this

Received by the editors April 22, 1992 and, in revised form, July 26, 1992.

1991 Mathematics Subject Classification. Primary 11B25, 33A15. 
is the well-known identity

$$
\sin (m z)=2^{m-1} \sin (z) \sin \left(z+\frac{\pi}{m}\right) \cdots \sin \left(z+\frac{(m-1) \pi}{m}\right) .
$$

In [1] Fraenkel proves that Raabe's identity for the Bernoulli polynomials,

$$
B_{n}(m z)=m^{n-1}\left(B_{n}(z)+B_{n}\left(z+\frac{1}{m}\right)+\cdots+B_{n}\left(z+\frac{m-1}{m}\right)\right),
$$

can be generalized to exact covers. (See also Beebee [3].) This last identity is an additive analogy to the Gauss-Legendre multiplication formula,

$$
(2 \pi)^{(m-1) / 2} m^{1 / 2-m z} \Gamma(m z)=\Gamma(z) \Gamma\left(z+\frac{1}{m}\right) \cdots \Gamma\left(z+\frac{m-1}{m}\right) .
$$

Evidently, these identities belong to a class which can be generalized to exact covers. For example, the referee has nominated the $q$-gamma function to this class (see [4]). The definition of this class and the common characteristics of the functions need to be determined.

Theorem 1. If $\Gamma(z)=g(z) \prod_{i=1}^{m} \Gamma\left(\left(z+a_{i}\right) / b_{i}\right)$ where $g$ has no zeros at the nonpositive integers and is defined for all complex $z$, then the set of $A P$ 's $C=$ $\left\{Z_{a_{i} b_{i}}: 1 \leq i \leq m\right\}$ is an exact cover with standardized offsets; and conversely if $C$ is an exact cover with standardized offsets, then

$$
\Gamma(z)=\frac{\Gamma\left(z / b_{1}\right)}{b_{1}{ }^{1-z / b_{1}}} \prod_{i=2}^{m} \frac{\Gamma\left(\left(z+a_{i}\right) / b_{i}\right)}{b_{i}^{-z / b_{i}} \Gamma\left(a_{i} / b_{i}\right)} .
$$

Proof. Suppose $\Gamma(z)=g(z) \prod_{i=1}^{m} \Gamma\left(\left(z+a_{i}\right) / b_{i}\right)$. The gamma function has the nonpositive integers for its only poles, and these poles have order 1 . Then $-a_{i}$ is a pole of the function on the right and hence of the function on the left. Thus $-a_{i}$ is a nonpositive integer, so $a_{i}$ is a nonnegative integer. If $n$ is a nonnegative integer, then $-a_{i}-n b_{i}$ is a pole on the right and hence on the left. Thus $a_{i}+n b_{i}$ is a nonnegative integer. Thus $b_{i}$ is a positive integer. If $\mathbf{n}$ is a nonnegative integer, $-n$ is a pole of order 1 on the left, and hence $\left(-n+a_{i}\right) / b_{i}$ is a pole for precisely one $i$ on the right and thus is a nonpositive integer: $\left(-n+a_{i}\right) / b_{i}=-m$. Hence $n=a_{i}+m b_{i}$ for each nonnegative $n$, so each nonnegative integer belongs to exactly one AP $Z_{a_{i} b_{i}}$. For finite $m$, this means $C$ is an exact cover. (There are infinite saturated systems of disjoint AP's that cover the nonnegative integers but not the integers. See Example 3 below.) Zero is a pole of the left side, so $\left(0+a_{i}\right) / b_{i}$ is a pole on the right for some $i$ and hence $a_{i} / b_{i}$ is a nonpositive integer for some $i$. But $a_{i} / b_{i} \geq 0$, so $a_{i}=0$ for some $i$. Suppose $a_{i} \geq b_{i}$. Then $b_{i}-a_{i} \leq 0$. Hence $b_{i}-a_{i}$ is a pole on the left. But this implies it is a pole on the right. Hence $\left(b_{i}-a_{i}+a_{j}\right) / b_{j}=-n$, for some nonnegative $n$. If $j=i$, this implies 1 is a pole of the gamma function, so $j \neq i$. Thus $a_{i}-b_{i}=a_{j}+n b_{j}$, which is a contradiction, because $a_{i}-b_{i}$ cannot belong to two AP's in the exact cover. Hence $0 \leq a_{i}<b_{i}$, so $C$ has standardized offsets.

Now suppose $C$ is an exact cover with $a_{1}=0,0 \leq a_{i}<b_{i}$. The referee suggested the following derivation of (2). It is similar to the derivation of (1) in Rainville [5] or Marsden [6]. Let $(z)_{n}=z(z+1) \cdots(z+n-1)$ and $N=$ 
a multiple of all the moduli, $b_{i}$. Since $C$ is an exact cover with standardized offsets,

$$
\{0,1,2, \ldots, N-1\}=\bigcup_{i=1}^{m}\left\{a_{i}+n b_{i}: 0 \leq n \leq N / b_{i}-1\right\}
$$

Thus

$$
\begin{aligned}
(z)_{N} & =\prod_{i=1}^{m}\left(z+a_{i}\right)\left(z+a_{i}+b_{i}\right) \cdots\left(z+a_{i}+\left(\frac{N}{b_{i}}-1\right) b_{i}\right) \\
& =\prod_{i=1}^{m} b_{i}^{N / b_{i}}\left(\frac{z+a_{i}}{b_{i}}\right)_{N / b_{i}} .
\end{aligned}
$$

By Theorem 9 of $[5],(z)_{n}=\Gamma(z+n) / \Gamma(z)$. Thus

$$
\frac{\Gamma(z+N)}{\Gamma(z)}=\prod_{i=1}^{m} b_{i}^{N / b_{i}} \frac{\Gamma\left(\left(z+a_{i}\right) / b_{i}+N / b_{i}\right)}{\Gamma\left(\left(z+a_{i}\right) / b_{i}\right)} .
$$

By Lemma 7 of [5], $\lim _{n \rightarrow \infty}\left((n-1) ! n^{z} / \Gamma(z+n)\right)=1$. Rearranging (3) and using this,

or

$$
\frac{\Gamma(z)}{\prod_{i=1}^{m} \Gamma\left(\left(z+a_{i}\right) / b_{i}\right)}=\lim _{N \rightarrow \infty} \frac{(N-1) ! N^{z}}{\prod_{i=1}^{m} b_{i}^{N / b_{i}}\left(N / b_{i}-1\right) !\left(N / b_{i}\right)^{\left(z+a_{i}\right) / b_{i}}},
$$

$$
\frac{\Gamma(z)}{b_{i}^{z / b_{i}} \prod_{i=1}^{m} \Gamma\left(\left(z+a_{i}\right) / b_{i}\right)}=\lim _{N \rightarrow \infty} \frac{(N-1) !}{N^{(m-1) / 2} \prod_{i=1}^{m} b_{i}^{\left(N-a_{i}\right) / b_{i}}}=\text { const }
$$

because $\sum 1 / b_{i}=1$ and $\sum a_{i} / b_{i}=(m-1) / 2$. Taking $\lim _{z \rightarrow 0}$ on the left, we see that

$$
\text { const }=\left(b_{1} \prod_{i=2}^{m} \Gamma\left(\frac{a_{i}}{b_{i}}\right)\right)^{-1} \text {. }
$$

Substituting this value of the constant in (4) yields (2). Neither this or any other proof that I have found applies to infinite exact covers.

Example 1. The Gauss-Legendre multiplication formula is a special case of (2). Proof. It is easy to see that $C_{m}=\left\{Z_{i-1, m}: 1 \leq i \leq m\right\}$ is an exact cover. In [2] I proved

Lemma. If $C=\left\{Z_{a_{i} b_{i}}: 1 \leq i \leq m\right\}$ is an exact cover then

$$
\prod_{i=2}^{m} \sin \pi \frac{a_{i}}{b_{i}}=\frac{b_{1}}{2^{m-1}}
$$

When we apply this lemma to the exact cover $C_{m}$, we get

$$
\prod_{i=2}^{m} \sin \pi\left(\frac{i-1}{m}\right)=\frac{m}{2^{m-1}}
$$

But $\Gamma(z) \Gamma(1-z)=\pi /(\sin \pi z)$. Hence

$$
\prod_{i=2}^{m} \Gamma\left(\frac{i-1}{m}\right) \Gamma\left(1-\frac{i-1}{m}\right)=\prod_{i=2}^{m} \frac{\pi}{\sin \pi((i-1) / m)}=\frac{(2 \pi)^{m-1}}{m}
$$


But $\prod_{i=2}^{m} \Gamma\left(\frac{i-1}{m}\right)=\prod_{i=2}^{m} \Gamma\left(1-\frac{i-1}{m}\right)$, so

$$
\prod_{i=2}^{m} \Gamma\left(\frac{i-1}{m}\right)=\frac{(2 \pi)^{(m-1) / 2}}{\sqrt{m}} \text {. }
$$

Now substitute $m z$ for $z, i-1$ for $a_{i}$, and $m$ for $b_{i}$ in (2), and use (5):

$$
\Gamma(m z)=\frac{\Gamma(z) \Gamma(z+1 / m) \cdots \Gamma(z+(m-1) / m)}{(2 \pi)^{(m-1) / 2} m^{1 / 2-m z}} .
$$

Rearrangement of this gives the Gauss-Legendre multiplication formula (1).

Example 2. As an example of (2), consider the exact cover

$$
C=\left\{Z_{04}, Z_{24}, Z_{16}, Z_{36}, Z_{56}\right\} \text {. }
$$

Substituting in (2) yields

$$
\Gamma(z)=\frac{\Gamma\left(\frac{z}{4}\right) \Gamma\left(\frac{z+2}{4}\right) \Gamma\left(\frac{z+1}{6}\right) \Gamma\left(\frac{z+3}{6}\right) \Gamma\left(\frac{z+5}{6}\right)}{4^{1-z / 4} 4^{-z / 4} 6^{-z / 6} 6^{-z / 6} 6^{-z / 6} \Gamma\left(\frac{2}{4}\right) \Gamma\left(\frac{1}{6}\right) \Gamma\left(\frac{3}{6}\right) \Gamma\left(\frac{5}{6}\right)} .
$$

For $z=10$, this equation is

$$
362,880=\frac{(1.329)(2)(.941)(1.082)(1.329)}{4^{-1.5} 4^{-2.5} 6^{-1.667} 6^{-1.667} 6^{-1.667}(1.772)(5.566)(1.772)(1.129)} .
$$

Example 3. $C_{n}=\left\{Z_{02} ; Z_{14} ; Z_{3,8} ; \ldots ; Z_{2^{n-1}-1,2^{n}} ; Z_{2^{n}-1,2^{n}}\right\}$ is an exact cover, the Grey cover, with $n+1$ AP's. Substitute $C_{n}$ into (2).

$$
\Gamma(z)=\frac{\Gamma(z / 2)}{2^{1-z / 2}} \frac{\Gamma\left(\left(2^{n}-1+z\right) / 2^{n}\right)}{\left(2^{n}\right)^{-z / 2^{n}} \Gamma\left(\left(2^{n}-1\right) / 2^{n}\right)} \prod_{i=2}^{n} \frac{\Gamma\left(\left(2^{i-1}-1+z\right) / 2^{i}\right)}{\left(2^{i}\right)^{-z / 2^{i}} \Gamma\left(\left(2^{i-1}-1\right) / 2^{i}\right)} .
$$

Taking $\lim n \rightarrow \infty$ on both sides we see that the product converges and

$$
\Gamma(z)=\frac{\Gamma(z / 2)}{2^{1-z / 2}} \frac{\Gamma(1)}{2^{0} \Gamma(1)} \prod_{i=2}^{\infty} \frac{\Gamma\left(1 / 2+(z-1) / 2^{i}\right)}{2^{-i z / 2^{i}} \Gamma\left(1 / 2-1 / 2^{i}\right)} .
$$

But the set of disjoint AP's $C=\left\{Z_{02} ; Z_{14} ; Z_{3,8} ; \ldots ; Z_{2^{n-1}-1,2^{n}} ; \ldots\right\}$ is not an infinite saturated exact cover, even though $\sum_{i=1}^{\infty} 1 / b_{i}=1$, because it does not cover -1 . Thus for $m=\infty$ (2) can hold, but $\mathrm{C}$ is not an exact cover. The proof of Theorem 1 made use of $\sum_{i=1}^{m} 1 / b_{i}=1$, so I speculate that if $\mathrm{C}$ is an unsaturated infinite exact cover, (2) does not hold. But if $\mathrm{C}$ is a saturated infinite exact cover, then (2) does hold. Limited numerical experiments support this conjecture.

Example 4. Define

$$
\Gamma_{a b}(z)= \begin{cases}\frac{\Gamma(z / b)}{b^{1-z / b}} & \text { if } a=0 \\ \frac{\Gamma((z+a) / b)}{b^{-z / b} \Gamma(a / b)} & \text { if } a \neq 0\end{cases}
$$

With this notation, (2) is

$$
\Gamma(z)=\prod_{i=1}^{m} \Gamma_{a b}(z) .
$$


The function $\Gamma_{a b}(z)$ has about the same relation to the AP $Z_{a b}$ that $\Gamma(z)$ has to the integers.

(a) $\Gamma(z)$ has its poles at the nonpositive integers. $\Gamma_{a b}(z)$ has its poles at the nonpositive integers in $Z_{a b}$.

(b) For $n \geq 1, \Gamma(1+z)=z \Gamma(z)$, and $\Gamma(n)=n-1$ !.

For the functions $\Gamma_{a b}(z)$, we have

$$
\begin{array}{ccccc}
\Gamma_{0 b}(b)=1 & \text { and } & \Gamma_{0 b}(n b) & =(n-1) b \cdot(n-2) b \cdots(2 b)(b) \\
\Gamma_{a b}(b)=a & \text { and } & \Gamma_{a b}(n b) & =(a+(n-1) b) \cdot(a+(n-2) b) \\
& & & \cdots(a+2 b)(a+b)(a) .
\end{array}
$$

Let $C$ be an exact cover, and let $z=M=\operatorname{lcm}\left\{b_{i}\right\}$. Then (6) is the self-evident formula

$$
\begin{aligned}
M-1 != & \left(\left(\frac{M}{b_{1}}-1\right) b_{1}\right)\left(\left(\frac{M}{b_{1}}-2\right) b_{1}\right) \cdots 2 b_{1} b_{1} \\
& \cdot \prod_{i=2}^{m}\left(a_{i}+\left(\frac{M}{b_{i}}-1\right) b_{i}\right)\left(a_{i}+\left(\frac{M}{b_{i}}-2\right) b_{i}\right) \cdots\left(a_{i}+b_{i}\right) a_{i} .
\end{aligned}
$$

\section{REFERENCES}

1. Aviezri S. Fraenkel, A characterization of exactly covering congruences, Discrete Math. 4 (1973), 359-366.

2. John Beebee, Some trigonometric identities related to exact covers, Proc. Amer. Math. Soc. 112 (1991), 329-338.

3. $\_$, Bernoulli numbers and exact covering systems, Amer. Math. Monthly 99 (1992), 946-948.

4. George Gasper and Mizan Rahman, Basic hypergeometric series, Cambridge Univ. Press, Cambridge, 1990.

5. Earl D. Rainville, Special functions, Macmillan, New York, 1960.

6. Jerrold E. Marsden, Basic complex analysis, Freeman, San Francisco, 1973.

7. B. Novák and S. Znám, Disjoint covering systems, Amer. Math. Monthly 81 (1974), 42-45.

8. Sherman K. Stein, Unions of arithmetic sequences, Math. Ann. 138 (1958), 289-294.

9. M. A. Berger, A. Felzenbaum, A. S. Fraenkel, and R. Holzman, On infinite and finite covering systems, Amer. Math. Monthly 98 (1991), 739-742.

Department of Mathematical Sciences, University of Alaska, Anchorage, Alaska

E-mail address: af jcb@alaska 